\title{
Comment and Analysis on Characteristics of Nano Alumina Materials in Different Dye-Sensitized Solar Cells
}

\author{
Yu-Feng Lin ${ }^{1,2 *}$, Hsin-Chieh Liu ${ }^{3}$, Chia-Hui Lin ${ }^{4}$ \\ ${ }^{1}$ Department of Chemical and Materials Engineering, Lung Hwa University of Science and Technology, Taoyuan, TW. \\ ${ }^{2}$ Center of General Education, Chung Yuan Christiant University, Taoyuan, TW. \\ ${ }^{3}$ Department of Information Management, YuanZe University, Taoyuan, TW. \\ ${ }^{4}$ The Affiliated Zhongli Senior High School of National Central University (CLHS), Taoyuan, TW.
}

\begin{abstract}
The new light-emitting organic materials used in OLEDs[1,2] belong to a type of charge injection, and our team has conducted a series of fluorescence studies. The dye-sensitized solar cell is currently the most discussed technology. The equipment that converts light energy into electrical energy is certainly worth studying. Our research on nano porous anodic aluminum oxide (AAO) nanomaterials is consistent with related research, in which titanium dioxide acts as an electron transport layer and AAO acts as a template. When the two are combined, their new properties are due to the higher mechanical strength of $\mathrm{Al} 2 \mathrm{O} 3$ nanotubes and the electronic conductivity of $\mathrm{TiO} 2$ nanorods, which will increase the surface area of the DSSC anode to absorb dyes and enhance the anode The structural strength of the membrane. Finally, using different dyes, we noticed an increase in the efficiency of using AAO.
\end{abstract}

\section{INTRODUCTION}

Refer to most research[3] and data analysis, we using high purity aluminum (purity of $99 \%, 3.61 \mathrm{~cm}^{2}$ size) as the base material, and by means of the use of nearly $0.4^{\circ} \mathrm{C}$, $0.29 \mathrm{M}(2.69 \mathrm{wt} \%)$ solution of oxalic acid to conduct anodization for the preparation of the periodic ordered, cellular shaped porous structure of the honeycomb alumina template, the effect of anodized phosphate reaming time and voltage on the diameter of the holes is discussed. In the process of aluminum anodized becomes alumina film, with appropriate regulation of the concentration of $29 \%$ phosphoric acid reaming time, and with the voltage parameter, there will be re-adjustment of the alumina pore size and pore spacing, and the distance of pore structure and pore spacing will therefore become large, resulting in forming different aspect ratio of the holes, in order to create a range of different nanoscale holes, called nanostructures holes template. Then regular and neat porous AAO template will be obtained, and then it possible to observe the result of the growth of nanoholes via SEM scanning electron microscope. Dyesensitized refers to the phenomenon of electronic excitation by light from the transfer of the dye molecules to the semiconductor conduction band, and then returned by the surrounding electron oxidation state to provide supplementary dye process. Its battery works for the nano-porous structure adsorbed organic dye molecules, the use of an organic dye layer as the main light-absorbing layer, which absorbs light energy generated excitons, when electron-hole Coulomb interaction effects still need a low conduction band Lumo energy level of its semiconductor materials, as electron-hole separation medium, then the electrons injected along a wide band gap semiconductor difference of the conduction band, and then transfer to the semiconductor electrode via a conductive glass are collected via the outer loop export. Returning to the electrode after the use, and then the electron transfer to the electrolyte, the electrolyte in the final oxidation-reduction reaction, reduction of the dye before the loss of electrons, thus forming a complete loop, as shown in Figure.1. Semiconductor materials in general choice of the most widely used TiO2, mainly due to this material has good corrosion resistance and chemical stability, and the price is inexpensive, non-toxic. Here the study on the selection of the dye to Ru-complex (N3, N719, as shown in Figure.2.) based. DSSC in comparison to other types of solar cells, because of its relatively low cost of production, process easier, long life, has the advantage of its use.

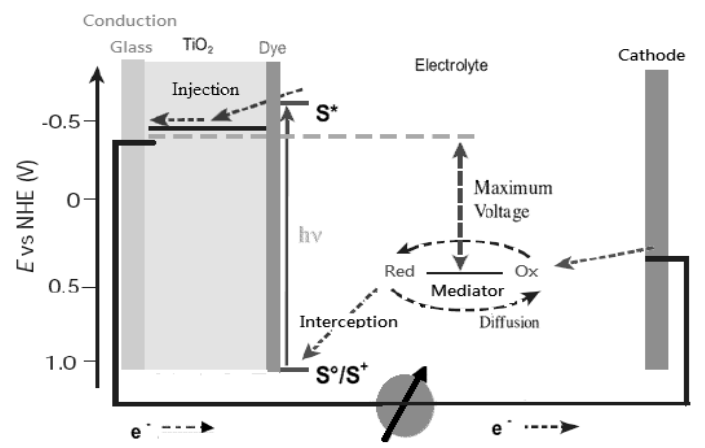

Figure.1. Mechanism of operation in the DSSC from. M.Grätzel (2004)

${ }^{*}$ Corresponding author: linlinphdyu@gmail.com 


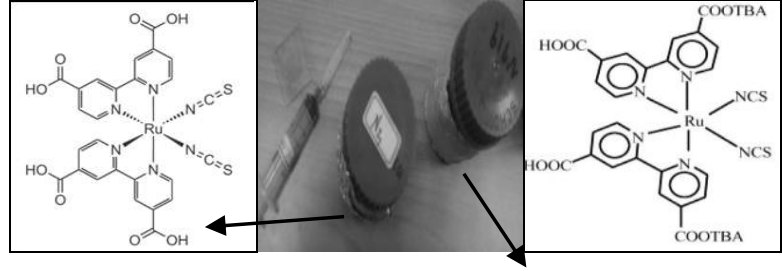

Figure.2. Dye to Ru-complex, N3 (left) and N719 (right) [4].

\section{EXPERIMENT}

\subsection{Mechanism}

After Keller et al. proposed the ideal porous alumina which is the composition of the hexagonal dense accumulation nest chamber. In-depth studies of the follow-up scholars believe that all types are similar to what is shown in Figure.6.3. The structure is divided into the upper layer of porous quality and the lower barrier layer of dense tissue. Under ideal conditions, each of the holes at the bottom of the barrier layer are half spherical with similar sphere radius and the size of the pore radius. The spacing between the nest chamber and pore size by means of the anode voltage and electrolyte types can be limited within a dozen nanometers to several hundred nanometers and it does not change over time but the thickness of the film will be a linear increase in relation with time. Because the formation of the pores is through the electric field assisted dissolution, a pore then has good collimation and good parallel to each other and perpendicular to the substrate surface. This array structure can be widely used in optoelectronics, thermal, mechanical and magnetic applied materials.

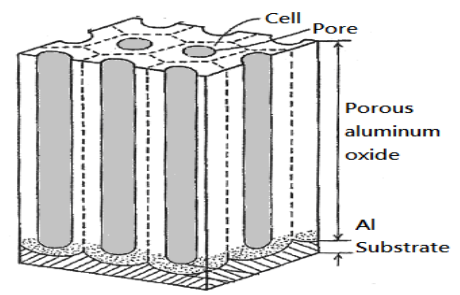

Figure.3. ideal porous holes of alumina structure.[5]

As shown in Figure.1., the sensitizer attached to the surface of a porous film absorbs the energy from sunlight (hv). The injection of electrons into the conduction band of the oxide is induced by the process of photo-excitation of the dye. Subsequently, the electron from electrolyte regenerates to the dye on the surface of porous films. The electrolyte contains the negative ion of iodide/triiodide $\left(\mathrm{I}^{-} / \mathrm{I}^{-}\right)$couple as a red-ox couple. Reduction of positive ion of dye $\left(\mathrm{S}^{+}\right)$by iodide $\left(\mathrm{I}^{-}\right)$regenerates the original form of the dye (So) while producing triiodide ions ( $\left.\mathrm{I}^{-}\right)$. This stops any significant increase of $\mathrm{S}^{+}$, which could recapture the conduction band electron at the surface. The iodide is regenerated by the reduction of the triiodide ions at the counter-electrode, where the electrons are acquired from the external circuit through the Pt. The whole reaction can be represented by following processes.[6,7]

$$
\begin{gathered}
\text { Anode: } \mathrm{So}+\mathrm{hv} \rightarrow \mathrm{S}^{*} \quad \text { Absorption (1) } \\
\mathrm{S}^{*} \rightarrow \mathrm{S}^{+}+\mathrm{e}^{-}(\mathrm{TiO} 2) \quad \text { Electron Injection }(2) \\
2 \mathrm{~S}++3 \mathrm{I}^{-} \rightarrow 2 \mathrm{So}+\mathrm{I}^{-} \quad \text { Regeneration (3) } \\
\text { Cathode: } \mathrm{I}^{-}+2 \mathrm{e}^{-}(\mathrm{Pt}) \rightarrow 3 \mathrm{I}^{-} \\
\text {Cell: } \mathrm{e}^{-}(\mathrm{Pt})+\mathrm{hv} \rightarrow \mathrm{e}^{-}(\mathrm{TiO} 2)
\end{gathered}
$$

Where the So represents the dye, the $S^{*}$ indicates the negative ion of dye. Thus, the device is generating electricity from light.

\subsection{Materials \& General Procedure of Fabrication}

The aluminum metal sheet of $99 \%$ purity is prepared (annealing at $390{ }^{\circ} \mathrm{C}$ for 2 hours), its size of $19 \mathrm{~mm} \times 19$ $\mathrm{mm}$ and a thickness of $185 \mu \mathrm{m}$ high purity aluminum sheet whose surface is wiped with acetone and the ethanol to remove impurities, and cleaned by DI-water in sonication. Assembly process: Firstly, place O-ring on the top of a groove to prevent the electrolyte from going into the specimen; place the aluminum specimen and aluminum spacers in sequence; then adhere the base mold to superstructure (as shown in Figure.4 and Figure.5). Then, conduct the electrolytic polishing; the objective is to make the surface of the test piece flat. The procedures are as follows: a beaker with a 1:5 per chloric acid and anhydrous ethanol solution as the electrolyte which is placed in a heated tank; in the electrolytic polishing liquid, aluminum specimen connected to positive, and a graphite rod connected to negative pole, the two apart from each other for $6 \mathrm{~cm}$, at the temperature of $30^{\circ} \mathrm{C}, 20 \mathrm{~V}$ DC current, electro-polishing for 35 minutes. After electrolytic polishing was the specular aluminum substrate. If the required electrolytic polishing aluminum substrate is more pure, the object reflectance is close at $100 \%$ after being polished, and the Al substrate surface will be flat. Anodization: place the polished aluminum test piece in a concentration of $0.29 \mathrm{M}$ phosphoric acid aqueous solution with the temperature controlled at $0.4^{\circ} \mathrm{C}$, stirred with a magnetic stir under an applied voltage of $180 \mathrm{~V}$ at $0{ }^{\circ} \mathrm{C}$ for $2 \mathrm{hrs}$. Use a mixed solution of $\mathrm{CuCl} 2$ and $\mathrm{HCl}$ at $35 \mathrm{wt} \%$ for the removal of the remaining aluminum beneath the barrier layer. There will be opalescent AAO film after the drying process as shown in Figure.6.

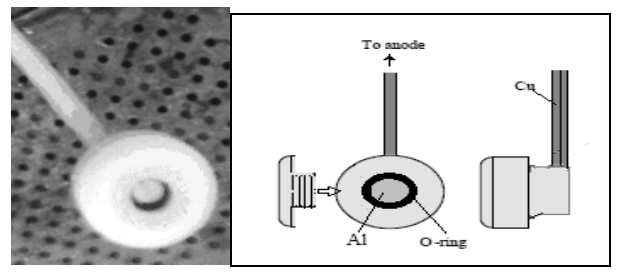

Figure.4. mounted to the aluminum mold, the front and side. 


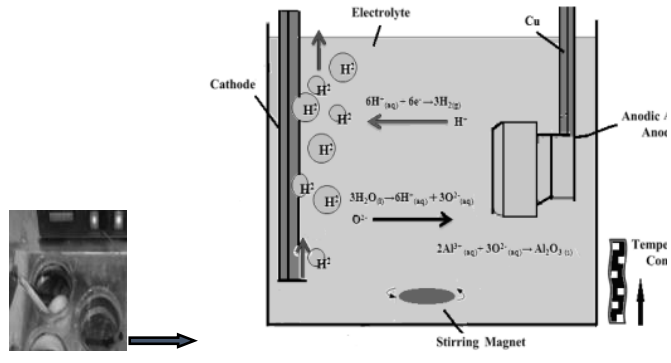

Figure.5. Electro polishing reaction process. Top view (left) and cross sectional view (right)
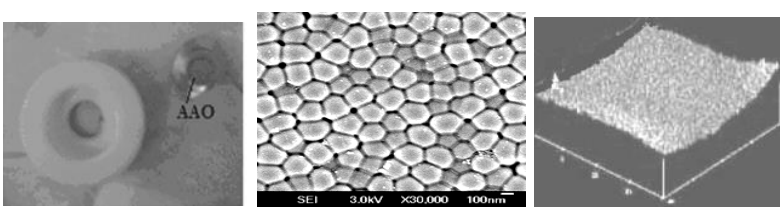

Fig.6. AAO membrane (left), SEM image (middle) AAO, and the smooth AAO under the AFM(right).

Furthermore, research DSSC, prepared by Sol-Gel method (Figure.7.), Photovoltaic measurements employed an AM 1.5 solar simulator equipped with a $150 \mathrm{~W}$ Xenon lamp, as shown in Figure.8. The power conversion efficiency (PCE, $\zeta$ ) of the DSSC is determined by the following equation:

$$
\zeta=\mathrm{Voc} \cdot \mathrm{Ff} \cdot \mathrm{J} / \mathrm{Is}
$$

where the $J$ is the photocurrent density measured at the situation of short circuit, the Voc is open-circuit photovoltage, Is is the intensity of the incident light, and Ff is the fill factor of the cell and equal to (Jmax $\cdot V \max )$ /(J・Voc). The maximum power (Pmax) is equal to $(\mathrm{Jmax} \cdot \mathrm{Vmax})$ at where the power output of the cell is maximal.

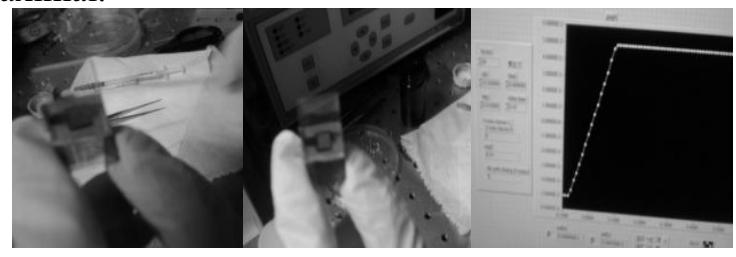

Figure.7. produced and measured DSSC(N719) device.
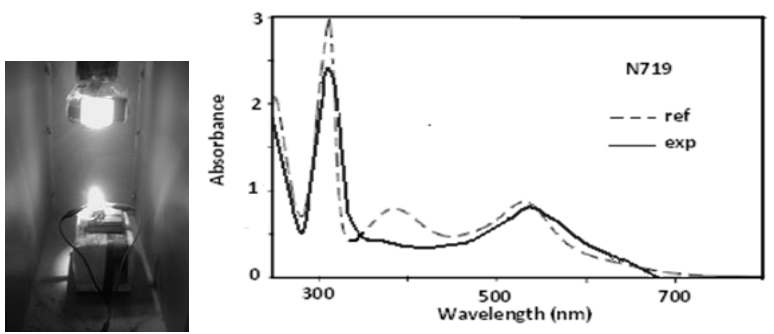

Figure.8. under150 W Xenon lamp(left) and got absorbance of DSSC(right).

\section{Results AND Discussion}

Second time anodic treatment to the pattern of the aluminum substrate: remove the film formed during the first time of anodization. Due to the barrier layer, there will be regular pattern of dents on the aluminum surface with the removal of the alumina layer. This pattern may be used as the initial core of anodic alumina hole membrane to help generation of holes during the second anodization. In this way, regular pattern of anodized aluminum film holes are gained. The electrolyte of $25^{\circ} \mathrm{C}$, $29 \mathrm{wt} \%$ phosphoric acid solution, H3PO4, is utilized to control a voltage of 30 volts to conduct the second anode processing for more than 70 mins. Then $0.25 \mathrm{M} \mathrm{NaOH}$ is used to remove the patterned barrier layer surface, (Figure.9) modify the barrier-layer surface and use $0.29 \mathrm{M}$ phosphate for a pore-widening process for $60 \mathrm{mins}$. The obtained AAO SEM microscopy images are shown in Figure.10. The phosphorylation reaming time changes after being anodized, so that the alumina pores grow into different sizes, as shown in Figure.10. and Figure.11.

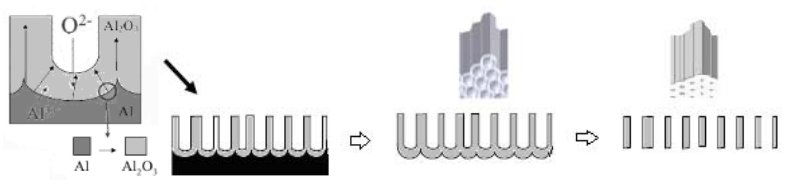

Figure.9. After secondary anodized process (the right picture shows the etching penetrates and becomes nano-column). [8,9]

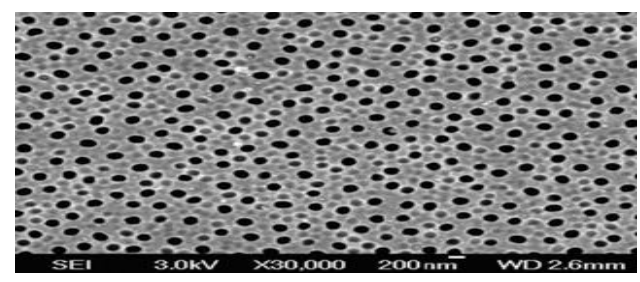

Figure.10. Initial reaming SEM (top view)[7]

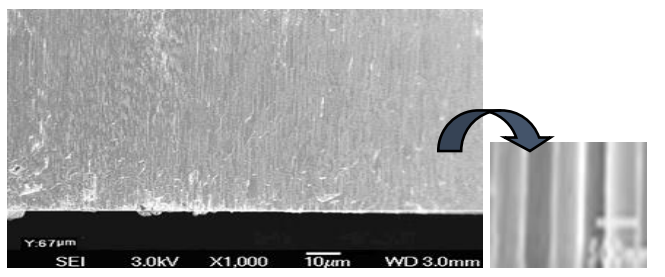

Figure.11. SEM image after widening (Cross-sectional view[8])

The relationship of Anode process time and voltage is shown in Figure.12.(A). In the process of anodization, owing to the current of $55 \mathrm{~mA}( \pm 5 \%)$, the relative voltage is unstable (not linear because of prolonged time). Holes slightly cone columnar $\mathrm{Al} 2 \mathrm{O} 3$ at $180 \mathrm{~V}$ and its is shown in Figure.12.(B), long as about the 350 to $400 \mathrm{~nm}$ rooms (see Figure.13.symbols ), This result conforms to AP Li, F. Muller, A. Briner's statement that the holes of the alumina template will be the largest in phosphoric acid electrolyte, and with the optimum voltage of 190V[9]. The diameter of hole is about $420 \mathrm{~nm}$. In addition to voltage, aperture gap is also affected by electrolyte concentration. If the time is prolonged, the hole will continue to increase into aluminum wire Figure.12.(C) (due to its strength, there is from other related AAO research in artificial bones). 


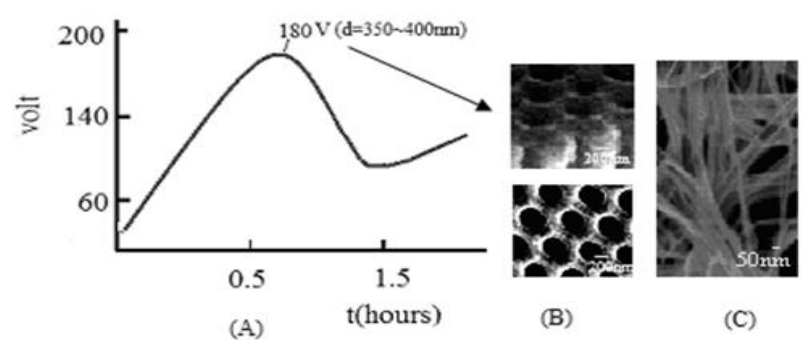

Figure.12. Anode process time and voltage is shown in Fig (A); side and front of the cone-cylinder, visible maximum aperture 350 to $400 \mathrm{~nm}$ shown in (B), too much erosion becomes filamentous (diameter50nm) and perforated Fig (C)[10].

The mentioned anode treating in making nano alumina column has an advantage because of its simple preparation, convenience and low cost. Its application in DSSC can lead to higher photo-efficiencies. In recent years, the research team at NCHU has made use of appropriate etching which does not result in the penetration of back-barrier layer (the barrier-layer side of AAO nanotube) to the form alumina nanotubes (shown as in the middle part of Figure.9, below is as semi-sphere). Make use of RF magnetron sputtering technology, sputter on ITO (indium tin oxide) thin film barrier structure at the back of the dome and improve the ITO lattice structure and conductivity by using the annealing process. Then, the ITO nanostructures electrode array with light transparency and good electrical conductivity are prepared to replace the thin film electrode structure. TiO2 (Titanium Dioxide) and dye is filled between the electrodes in order to develop more efficient DSSC. Dr.Wang and co-workers produced high-performance DSSC nano-structured electrode board (Figure.14.(A)). After verification of optical flow measurement, the result shows that the conversion efficiency is up to $0.12 \%$. Dr.Ko's team(@ CTU) made use of anodic aluminum oxide template to assemble hollow $\mathrm{TiO} 2$ hemispheres under the porous alumina with the $\mathrm{TiO} 2$ nanotubes inside applied in DSSC as dye sensitized solar cell electrode, of which the efficiency is 1.7 times the conventional electrode cell, Figure.14.(B).

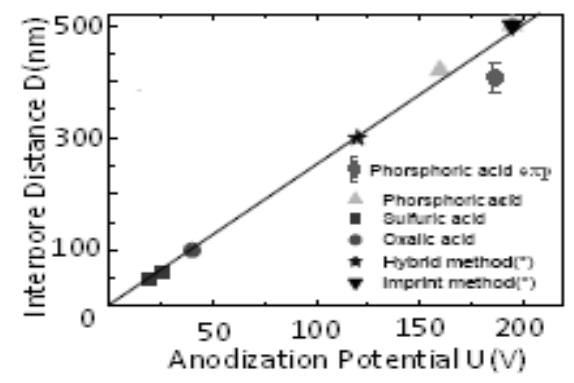

Figure.13. Holes slightly cone columnar Al2O3 at 180V (see symbols from our exp)[10]

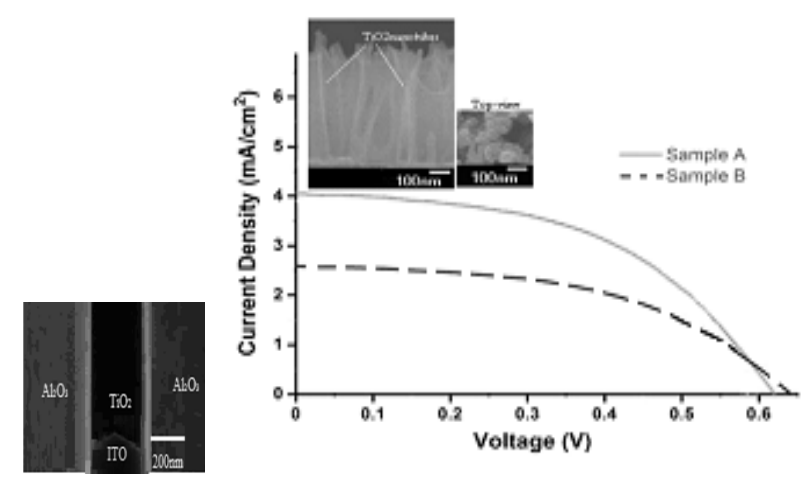

Figure.14.(A) TiO2/ITO/AAO, nano-array electrode SEM image; (B) $\mathrm{TiO} 2$ film under porous alumina with $\mathrm{TiO} 2$ nanotubes inside (Sample A), typical $\mathrm{TiO} 2$ nanoparticles (Sample B). Photovoltaic measurements employed an AM 1.5 solar simulator equipped with a $150 \mathrm{~W}$ Xenon lamp, and the power of the simulated light was calibrated to $100 \mathrm{~mW} / \mathrm{cm}^{2}$. The photocurrent (I) and photo-voltage (V) of the cell were measured with an active area of $0.25 \mathrm{~cm}^{2}$ [11].

\section{CONCLUSION}

1). This uses a way to actually produce anodic alumina membrane pores ( 350 to $400 \mathrm{~nm}$ ) and nano cone column. Porous material means the material that has many regular array of holes. It can serve as a template by using anodic aluminum oxide film[12] to prepare a regular array (nanopore arrays) nanostructure, such as nano-electrodes filled column, nanofibers which have the advantages of high hardness, wear resistance. Related studies have confirmed that AAO has a huge surface area when used in DSSC technically so that they become excellent adsorbent to profit dye adsorption. And because of the formation of neat cone, the optical refractive will reduce and is good for light absorption. In addition, studies have pointed out that template assisted deposition offers flexibility in terms of material and dimensional control [13].

2). $\mathrm{TiO} 2$ nanotubes generate along anodized aluminum wall, and the barrier along the anodized aluminum wall titanium can effectively prevent contact of $\mathrm{TiO} 2$ nanotubes which will delay electronic transmission, which proved to improve DSSC battery efficiency (1.7 times).

\section{ACKNOWLEDGMENT}

A part of this research was supported by "Translational medicine and agronomy personnel training Pioneer Program".

\section{REFERENCES}

1. Y.F Lin, P.W Cheng, S.H Chiu,C.H Wang, A.C Yeh, , Life Sci J,2012,9(3),409-412.

2. Y.F Lin, S.H Chiu,C.H Wang, A.C Yeh, C.H Wang, Advanced Materials Research,2012,557-559,748754. 
3. G. E Thompson.; G. C Wood, Treatise on Materials Science and Technology, Academic Press Inc. Ltd., ch5, 206 (1983).

4. Md.K. Nazeeruddin; R. Humphry-Baker; P. Liska; M.Grätzel, J. Phys. Chem. B, 2003, 107, 8981-8987.

5. D.Almawlawi; K.A Bosnick; A.Osika; M.Moskovits, Adv. Mater., 2000, 12, 1252.

6. M. Grätzel, Nature, 2001, 414, 338-344.

7. M. Grätzel, J. Photochem. Photobio. A,2004, 164, 314.

8. O.Jessensky; F. Muller; U. Gosele, Appl. Phys. Lett.,1998,72,1173-1175.

9. Y.Kanamoria, K.Hane, H.Sai;H. Yugami, Appl. Phys.Lett., 2001, 78.

10. A. P. Li, F. Muller, A. Birner, K. Nielsch, U. Gosele, J Appl. Phys, 1998, 84,6023.

11. C.C Ho, MD thesis at NTCU,2009,64.

12. A.M.Md Jani, D. Losic, N.H Voelcker, Progress in Materials Science ,2013,58(5),636-704.

13. A. Ganapathi, P. Swaminathan, and L. Neelakantan, Appl. Nano Mater, 2019, 2, 9, 5981-5988. 